\title{
Exploring Racial and Age Disproportionalities in COVID-19 Positive Pediatric Cohort
}

Emily Freeman ${ }^{1}$, Yiqiang Song ${ }^{1,2}$, Katie Allen ${ }^{2}$, Siu Hui ${ }^{1,2}$, Eneida Mendonca ${ }^{1,2}$

${ }^{1}$ Indiana University School of Medicine, ${ }^{2}$ Regenstrief Institute

Background: Social and health inequities place marginalized populations at increased risk of contracting the novel coronavirus 2019 (COVID-19). While COVID-19 literature continues to accumulate, there remains a lack of comprehensive epidemiological data on COVID-19 in children. The study aims to identify demographic trends in disease severity amongst COVID-19 positive pediatric patients.

Methods: We analyzed the medical records of 2217 laboratory-confirmed COVID-19 pediatric patients, ages 0-18, across Indiana. Working with Regenstrief Institute Center of Biomedical Informatics, data was extracted from the databases of Indiana Network for Patient Care, Indiana University Health, and Eskenazi Health from February $28^{\text {th }}, 2020$ to July $13^{\text {th }}, 2020$. Factors of interest were age, race, and ethnicity. The study assessed the clinical outcome of disease severity which was defined by one of the following clinical designations: outpatient management exclusively, emergency care without hospital admission, non-pediatric intensive care unit (PICU) hospitalization, PICU hospitalization, and death.

Results: The laboratory confirmed COVID-19 pediatric cohort was composed of $12.2 \%$ ( $\mathrm{N}=$ 270) Black or African American, 49.3\% ( $\mathrm{N}=1094)$ white, and 3.2\% ( $\mathrm{N}=71)$ American Indian/Alaska Native, Asian/Pacific Islander, and Multiracial combined group. 34.4\% of Black or African American patients required emergency (12.2\%) or inpatient care $(22.2 \%)$ while $24.4 \%$ white patients required emergency $(7.0 \%)$ or inpatient care (17.3\%). $17.6 \%$ of the cohort was 0 5 years old, $24.8 \%$ was $6-12$ years old, and $57.6 \%$ was $13-18$ years old. $30.9 \%$ of the $0-5$ age group required emergency or inpatient care while the percentages of the 6-12 age group and $13-18$ age group requiring emergency or inpatient care were $20.6 \%$ and $18.9 \%$, respectively.

\section{Conclusion:}

While our data is preliminary and requires additional validation, our exploration of racial and age disproportionalities in pediatric coronavirus severity serves to expand on the current COVID-19 literature and understanding of this virus.

Word Count: 290 